\title{
MAPEAMENTO DAS FORMAS DO RELEVO PARA ESTIMATIVA DE CUSTOS DE FERTILIZAÇÃO EM CANA-DE-AÇÚCAR
}

\author{
RODRIGO B. SANCHEZ ${ }^{1}$, JOSÉ MARQUES JÚNIOR ${ }^{2}$, GENER T. PEREIRA ${ }^{3}$, \\ JORGE BARACAT NETO ${ }^{4}$, DIEGO S. SIQUEIRA ${ }^{5}$, ZIGOMAR M. DE SOUZA ${ }^{6}$
}

\begin{abstract}
RESUMO: A modelagem matemática associada ao conhecimento da variabilidade dos atributos do solo e mapeamento das formas do relevo pode auxiliar no manejo da fertilidade do solo em usinas sucroalcooleiras. O presente trabalho teve como objetivo avaliar o uso da geoestatística e da modelagem matemática na estimativa de custos de fertilização, em diferentes formas do relevo. Em uma área de 200 ha, foram identificadas duas formas de relevo, uma côncava e outra convexa, sendo os solos coletados nos pontos de cruzamento de uma malha, com intervalos regulares de $50 \mathrm{~m}$, perfazendo um total de 623 pontos. As amostras foram submetidas a análises químicas, e, posteriormente, os dados foram avaliados por meio da estatística descritiva, geoestatística e modelagem matemática. Os resultados mostraram que, quando as formas do relevo são incorporadas às análises geoestatística e de modelagem matemática, ocorre aumento na eficiência de aplicação do calcário, fósforo e potássio no solo.
\end{abstract}

PALAVRAS-CHAVE: zonas de manejo específico, taxa variada, agricultura de precisão.

\section{MAPPING THE RELIEF FORMS TO ESTIMATE THE FERTILIZATION EXPENSES IN SUGARCANE}

\begin{abstract}
The mathematical model associated with the knowledge of the soil variability properties and mapping the relief forms can assist in the management of soil fertility in sugarcane companies. This study aimed to evaluate the use of geostatistics and mathematical modeling in the fertilization expense estimative in different relief forms. In an area of 200 ha has been identified two relief forms, one concave and one convex, and the soils collected was at the crossing points of a grid with intervals of $50 \mathrm{~m}$, a total of 623 points. The samples were subjected to chemical analysis and subsequently the data were analyzed using descriptive statistics, geostatistics and mathematical modeling. The results showed that when the relief forms are incorporated into the geostatistical analysis and mathematical modeling, there is an increase in the efficiency of limestone, phosphorus and potassium application in the soil.
\end{abstract}

KEYWORDS: specific areas of management, variable rates, precision agriculture.

\section{INTRODUÇÃO}

Uma das necessidades da agricultura moderna é a predição da variabilidade espacial dos atributos do solo em escalas mais detalhadas para o gerenciamento sustentável e otimização das práticas de manejo (KORSAETH \& RILEY, 2006). Neste enfoque, a agricultura de precisão é inserida com vistas ao aumento da rentabilidade das culturas por meio do aumento de produtividade e utilização racional de insumos (LAMBERT et al., 2006).

A hipótese básica da agricultura de precisão visa à otimização da aplicação de insumos com o objetivo de aumentar os lucros e diminuir a contaminação ambiental (KORSAETH \& RILEY,

\footnotetext{
${ }^{1} \mathrm{Eng}^{\mathrm{o}} \mathrm{Agr}^{\mathrm{o}}$, Diretor de Pesquisa e Desenvolvimento, Usina São Domingos, rodrigo@ usinasaodomingos.com.br.

${ }_{3}^{2}$ Faculdade de Ciências Agrárias e Veterinárias, UNESP, Departamento de Solos e Adubos, marques@ fcav.unesp.br.

${ }^{3}$ Faculdade de Ciências Agrárias e Veterinárias, UNESP, Departamento de Ciências Exatas, genertp@ fcav.unesp.br.

${ }^{4} \mathrm{Eng}^{\mathrm{o}}$ Agr ${ }^{\mathrm{o}}$, Usina São Domingos, jorge@ usinasaodomingos.com.br.

${ }_{6}^{5}$ Pós-graduando do PPG em Produção Vegetal, FCAV/ UNESP, diego_silvasiqueira@yahoo.com.br.

${ }^{6}$ Faculdade de Engenharia Agrícola, UNICAMP, Campinas - SP, zigomarms@ feagri.unicamp.br.

Recebido pelo Conselho Editorial em: 21-9-2009

Aprovado pelo Conselho Editorial em: 17-11-2011
} 
2006). Nesse aspecto, são realizadas aplicações localizadas em doses variadas na área, definidas a partir da identificação de zonas de manejo específico. A produtividade de grãos obtidas na colheita são altamente variáveis em uma determinada área, em consequência das interações complexas entre os diferentes fatores, tais como a topografia, as propriedades do solo e as práticas de manejo (KRAVCHENKO \& BULLOCK, 2002).

As relações solo-geomorfologia tornaram-se uma ferramenta importante para identificar e mapear zonas de manejo específico, que são regiões com máxima homogeneidade das propriedades do solo dentro da mesma área. A identificação dessas zonas garante a localização de limites mais precisos entre áreas distintas, permitindo que técnicas agronômicas possam ser transferidas com facilidade e economia para ambientes semelhantes (MALLARINO et al., 2001; BISHOP \& MCBRATNEY, 2002, SIQUEIRA et al., 2010a).

Pesquisas têm utilizado variáveis topográficas para explicar os padrões aleatórios da variação dos atributos do solo (SINGH \& KAR, 2001; LANDI et al., 2004) e do rendimento e qualidade de culturas agrícolas (KRAVCHENKO \& BULLOCK, 2002; SI \& FARRELL, 2004; MARTÍN et al., 2005). Neste contexto, melhor compreensão dos padrões espaciais das culturas, baseada em informações topográficas, pode fornecer aos agricultores oportunidades de aplicação de sítios específicos de manejo do solo ou aplicação de insumos a taxa variável (KASPAR et al., 2003).

Ressalta-se ainda a facilidade na obtenção de dados sobre relevo, quanto ao tempo e mão de obra consumidos, em comparação à mensuração de outras variáveis do solo (KRAVCHENKO \& BULLOCK, 2002). Portanto, a identificação das zonas de manejo específico pode ser uma solução prática e operacional para a adoção da agricultura de precisão. Alguns pesquisadores têm proposto a associação de diferentes métodos de mapeamento para a identificação destas zonas. Mais recentemente, pode-se citar o trabalho de SIQUEIRA et al. (2010a), que propõem o uso de conceitos da relação solo-geomorfologia associados a técnicas de análise geoestatística e multivariada para identificação de áreas com diferentes potenciais para a cultura da laranja. Dessa maneira, os limites estabelecidos no campo, baseados na conceituação de corpos naturais, são validados matematicamente.

Assim, as relações solo-geomorfologia, a geoestatística (WEBSTER, 2000; SOUZA et al., 2006) e os modelos matemáticos quantitativos de otimização operacional (IANNONI \& MORABITO, 2006; KAWAMURA et al., 2006; PAIVA \& MORABITO, 2007) são ferramentas que podem auxiliar na questão operacional agrícola do setor sucroalcooleiro. Desse modo, o objetivo deste trabalho foi avaliar o uso da geoestatística e da modelagem matemática na estimativa de custos de fertilização do solo em diferentes formas do relevo.

\section{MATERIAL E MÉTODOS}

\section{Características da área}

A área de estudo localiza-se no município de Catanduva, a noroeste do Estado de São Paulo, com coordenadas geográficas de $21^{\circ} 05^{\prime}$ de latitude sul e $49^{\circ} 01^{\prime}$ de longitude oeste. A região encontra-se na província geomórfica do Planalto Ocidental Paulista, tendo como material de origem as unidades geológicas pertencentes às rochas sedimentares do Grupo Bauru, representados pela formação Adamantina (IPT, 1981). O solo da área de estudo foi classificado como Argissolo Vermelho-Amarelo eutrófico, com textura média a argilosa (EMBRAPA, 2006).

Elaborou-se o modelo de elevação digital, utilizando os dados do levantamento planialtimétrico, que juntamente com as atividades de campo, possibilitou a identificação e a separação das formas do terreno e sua posterior classificação geomorfológica, conforme TROEH (1965). De acordo com esta classificação foram mapeadas duas formas do relevo, sendo uma côncava e a outra convexa (Figura 1). 


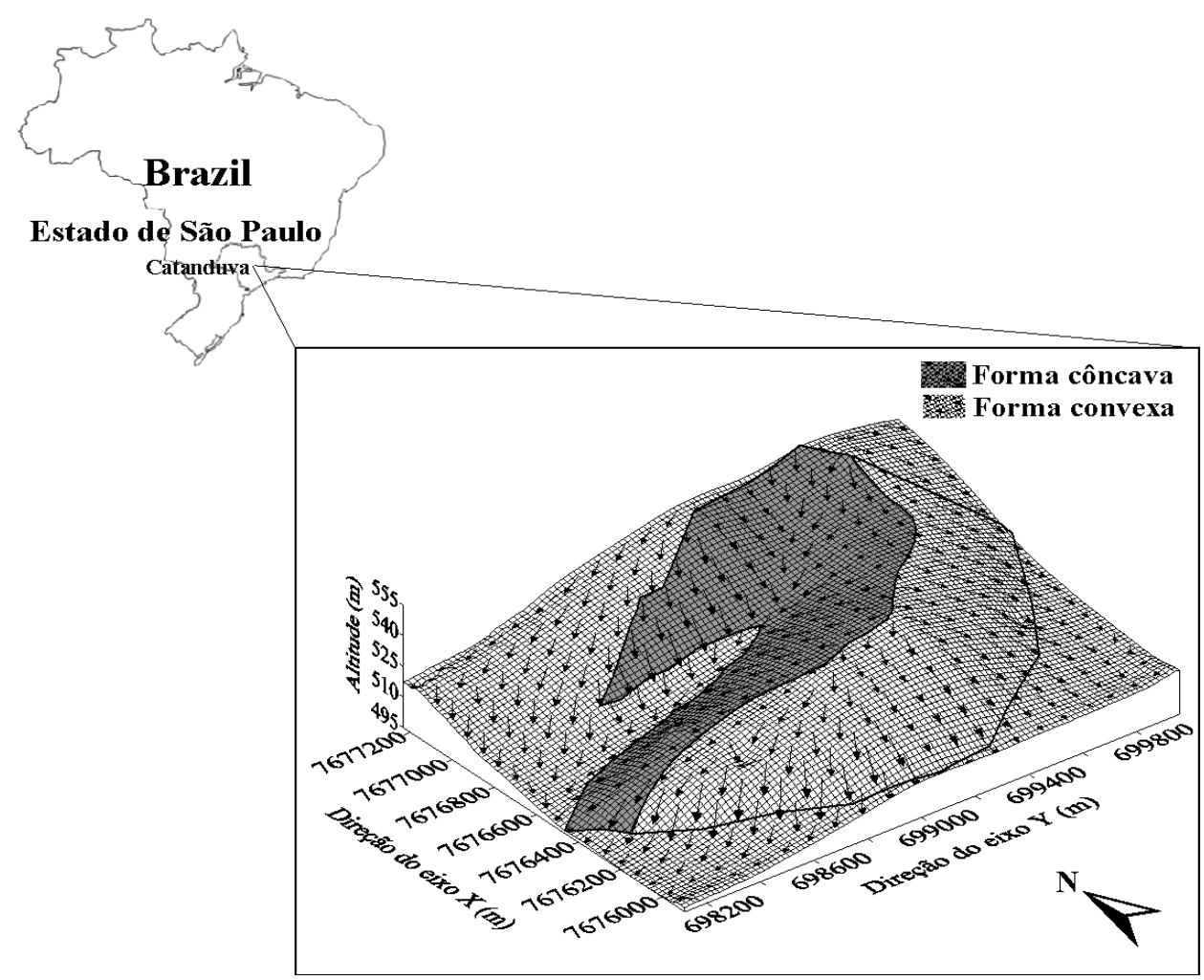

FIGURA 1. Localização da área, modelo digital de elevação do terreno (MDE), identificação das áreas côncava e convexa, e simulação do fluxo superficial de água. Location of the area, digital terrain elevation (DEM), identification of concave and convex areas and simulation of water flow surface.

A área de estudo possui 200 ha e apresenta um histórico de cultivo intensivo de cana-de-açúcar por mais de 20 anos. O histórico de manejo das duas áreas é idêntico, e a variedade de cana-de-açúcar plantada, em 2003, foi a SP-80-3250. A adubação líquida de plantio foi realizada com $812 \mathrm{~L} \mathrm{ha}^{-1}$ da fórmula comercial (04-12-10), sendo, na adubação de cana-soca, utilizada $944,21 \mathrm{~L} \mathrm{ha}^{-1}$ da fórmula comercial (10-00-10). Torta de filtro e vinhaça não foram aplicadas nas áreas.

\section{Coleta de amostras e avaliação dos atributos do solo}

$\mathrm{Na}$ área, os solos foram amostrados com trado nos pontos de cruzamento de uma malha, com intervalos regulares de $50 \mathrm{~m}$, na profundidade de 0,0-0,2 $\mathrm{m}$, perfazendo um total de 623 pontos.

$\mathrm{Na}$ caracterização química do solo, foram determinadas as bases cálcio $(\mathrm{Ca})$, magnésio $(\mathrm{Mg})$, potássio $(\mathrm{K})$ e o fósforo disponível (P), extraídos utilizando-se do método da resina trocadora de íons (RAIJ et al., 2001). Com base nos resultados das análises químicas, calculou-se a capacidade de troca de íons (CTC) e a saturação por bases (V\%).

As necessidades de calcário (NC), fósforo (NP) e potássio (NK) foram estimadas por meio da média dos 623 pontos e pela variabilidade espacial entre os pontos de amostragem. Para o cálculo da necessidade de calcário, utilizou-se a fórmula proposta por RAIJ et al. (1997), que considera a CTC e o nível de (V\%) atual do solo, que deve ser elevado até o valor ideal para a cultura, sendo, no caso da cana-de-açúcar no Estado de São Paulo, de 60\%, considerando-se PRNT de $100 \%$. Para o cálculo da adubação de fósforo e potássio, foram utilizadas as tabelas de adubação propostas por RAIJ et al. (1997) e considerando uma produtividade esperada de 100 a $150 \mathrm{t} \mathrm{ha}^{-1}$. 


\section{Análise geoestatística}

Os atributos químicos do solo foram avaliados por meio da análise estatística descritiva, utilizando-se do SAS (SCHLOTZHAVER \& LITTELL, 1997). A dependência espacial foi verificada por meio de ajustes de semivariogramas (VIEIRA et al., 1983; ROBERTSON, 1998), com base na pressuposição de estacionariedade da hipótese intrínseca, a qual é estimada por:

$$
\hat{\gamma}(\mathrm{h})=\frac{1}{2 \mathrm{~N}(\mathrm{~h})} \sum_{\mathrm{i}=1}^{\mathrm{N}(\mathrm{h})}\left[\mathrm{Z}\left(\mathrm{x}_{\mathrm{i}}\right)-\mathrm{Z}\left(\mathrm{x}_{\mathrm{i}}+\mathrm{h}\right)\right]^{2}
$$

em que: $\hat{\gamma}(h)$ é o valor da semivariância para uma distância $h$; $N(h)$ é o número de pares envolvidos no cálculo da semivariância; $\mathrm{Z}\left(\mathrm{x}_{\mathrm{i}}\right)$ é o valor do atributo do solo na posição $\mathrm{x}_{\mathrm{i}} ; \mathrm{Z}\left(\mathrm{x}_{\mathrm{i}}+\mathrm{h}\right)$ é o valor do atributo do solo separado por uma distância $\mathrm{h}$ da posição $\mathrm{x}_{\mathrm{i}}$. $\mathrm{O}$ semivariograma é representado pelo gráfico $\hat{\gamma}(h)$ versus $\mathrm{h}$. Do ajuste de um modelo matemático aos valores calculados de $\hat{\gamma}(h)$, são estimados os coeficientes do modelo teórico para o semivariograma (o efeito pepita, $\mathrm{C}_{0}$; patamar, $\mathrm{C}_{0}+\mathrm{C}_{1}$; e o alcance, a).

Para analisar o grau da dependência espacial dos atributos em estudo, utilizou-se a classificação de CAMBARDELLA et al. (1994), em que são considerados como de dependência espacial forte os semivariogramas que têm um efeito pepita $<25 \%$, moderada quando está entre 25 e $75 \%$ e fraca $>75 \%$ do patamar.

Os modelos de semivariogramas considerados foram o esférico, o exponencial, o linear e o gaussiano, sendo ajustados por meio do programa $\mathrm{GS}^{+}$(ROBERTSON, 1998) e, posteriormente, tais modelos foram usados no desenvolvimento de mapas de isolinhas (krigagem). Em caso de dúvida entre mais de um modelo, para o mesmo semivariograma, considerou-se o de maior valor do coeficiente de correlação, obtido pelo método de validação cruzada. Para a elaboração dos mapas de distribuição espacial das variáveis, foi utilizado o programa Surfer (GOLDEN SOFTWARE, 1999).

\section{Modelagem matemática}

A adubação da área em estudo foi submetida à modelagem matemática (MM), sendo que este modelo consiste em uma programação linear, desenvolvida pela Usina São Domingos Açúcar e Álcool S/A, para a estimativa das quantidades de insumos que devem ser aplicados na área.

O programa de otimização de custos, com base em modelos matemáticos, necessita de planilhas de entrada com as seguintes informações: área do lote a ser tratado, onde cada lote representa um conjunto de fazendas com mesma necessidade de correção do solo, variedade, tipo de ambiente de produção e idade do canavial; exigência em adubação por lote; custos de cada fertilizante cotado em moeda brasileira, e quantidades mínimas e máximas para aplicação de cada fertilizante.

No caso de utilizar a modelagem matemática em conjunto com a análise geoestatística, considerou-se cada ponto da malha em estudo como um lote, o qual representa uma área de 0,328 ha, sendo tratada de acordo com sua exigência em fertilidade determinada com base nos resultados das análises químicas para fósforo e potássio. $\mathrm{O}$ nitrogênio foi calculado com base na produtividade esperada. A cotação dos fertilizantes utilizados foi feita na cidade de Catanduva e realizada durante o mês de outubro de 2006. Para a adubação da área, excluíram-se, nas planilhas de entrada, as quantidades mínimas e máximas dos seguintes fertilizantes: torta de filtro, vinhaça e compostagem, uma vez que não foram aplicados.

Dois tipos de adubação foram otimizados neste programa matemático computacional. Um deles foi a adubação no sistema agrícola convencional (AC), que é adotado pela Usina São Domingos Açúcar e Álcool S/A, e consiste na aplicação do adubo líquido sob a fórmula comercial (04-12-10). O segundo sistema de adubação que foi otimizado, baseia-se nos conceitos de agricultura de precisão (AP) para aplicação adubo químico em taxa variada, que é função dos níveis de nutrientes para cada ponto da malha e produtividade esperada. 
Para a adubação convencional, os teores foram dose única de nitrogênio, fósforo e potássio (N-P-K), correspondente a 40; 120 e $100 \mathrm{~kg} \mathrm{ha}^{-1}$, respectivamente. Neste caso, toda a área foi considerada como um único lote (200 ha). Já na adubação em taxa variada, foi utilizada a necessidade de cada ponto amostrado. Com esses dados, o modelo matemático pôde simular as duas formas de adubação (AC e AP). Com resultado destas simulações, obtiveram-se as quantidades de fertilizantes e o custo em unidade monetária, por lote, a serem aplicadas. Os custos com os fertilizantes foram transformados para dólar (US\$), com um valor de câmbio de R $\$ 2,15$, contado no mês de setembro de 2006.

\section{RESULTADOS E DISCUSSÃO}

A média e a mediana das variáveis do solo, nas duas formas do relevo, foram similares. Isso indica que as variáveis analisadas possuem uma distribuição próxima à normal. Os valores dos coeficientes de curtose e assimetria próximos de zero confirmam esse resultado. Dessa maneira, não há limitações para a aplicação de técnicas geoestatísticas, com exceção apenas para o fósforo, nas duas formas de relevo (Tabela 1). O fósforo foi a variável que apresentou a distribuição mais próxima da assimétrica, concordando com os valores de assimetria e curtose. Nestes casos, existe a possibilidade de o valor médio não representar adequadamente esta variável na área, corroborando os resultados observados por CAMBARDELLA et al. (1994), em que a log-transformação dos dados reduziu a assimetria, porém a distribuição apresentou curtose, indicando maior falha na redução da curtose do que na assimetria.

Analisando os valores mínimos e máximos, notou-se grande amplitude para o fósforo e saturação por bases, com valores de fertilidade do solo variando nas faixas de muito baixa a muito alta (RAIJ et al., 1997). Essa amplitude de valores revela os problemas que podem ocorrer quando se utiliza a média como base para a tomada de decisão sobre o manejo químico em áreas agrícolas. Assim, em alguns locais, a aplicação de fertilizantes ou corretivos será abaixo da dose necessária, em outros, a aplicação será adequada, e, em alguns pontos haverá aplicação excessiva (DODERMANN \& PING, 2004).

TABELA 1. Resultados da estatística descritiva para os atributos químicos e necessidade de fósforo, potássio e calcário na profundidade de 0,00-0,20 m. Results of descriptive statistics for the chemical and the need for phosphorus, potassium and limestone at a depth of $0.00-0.20 \mathrm{~m}$.

\begin{tabular}{ccccccccc}
\hline Atributos & Área & Média & Mediana & Máximo & Mínimo & Assimetria & Curtose & C.V.\% ${ }^{(5)}$ \\
\hline $\begin{array}{c}\mathrm{P} \\
\left(\mathrm{mg} \mathrm{dm}^{-3}\right)\end{array}$ & Côncava & $25,2 \mathrm{a}$ & 14,0 & 103,0 & 3,0 & 1,4 & 1,1 & 104,0 \\
$\mathrm{~K}$ & Convexa & $24,2 \mathrm{a}$ & 11,0 & 147,0 & 3,0 & 2,0 & 3,6 & 123,4 \\
\hline$\left(\mathrm{mmol}_{\mathrm{c}} \mathrm{dm}^{-3}\right)$ & Côncava & $2,1 \mathrm{~b}$ & 2,0 & 4,5 & 0,8 & 0,6 & $-0,2$ & 40,6 \\
\hline $\mathrm{Ca}$ & Convexa & $2,5 \mathrm{a}$ & 2,3 & 6,1 & 0,8 & 0,9 & 0,6 & 42,7 \\
\hline$\left(\mathrm{mmol}_{\mathrm{c}} \mathrm{dm}^{-3}\right)$ & Côncava & $15,5 \mathrm{a}$ & 14,0 & 34,0 & 3,0 & 0,7 & 0,4 & 38,6 \\
\hline $\mathrm{Mg}$ & Convexa & $15,5 \mathrm{a}$ & 14,0 & 44,0 & 4,0 & 1,3 & 2,3 & 45,1 \\
\hline$\left(\mathrm{mmol}_{\mathrm{c}} \mathrm{dm}^{-3}\right)$ & Côncava & $6,2 \mathrm{a}$ & 6,0 & 13,0 & 1,0 & 0,6 & 0,3 & 35,9 \\
\multirow{2}{*}{$\mathrm{V}^{(1)}$} & Convexa & $4,4 \mathrm{~b}$ & 4,0 & 8,0 & 2,0 & 0,5 & $-0,2$ & 32,8 \\
\hline \multirow{2}{*}{$\mathrm{NP}^{(2)}$} & Côncava & $60,3 \mathrm{a}$ & 60,0 & 98,0 & 21,0 & 0,0 & 0,6 & 129,0 \\
& Convexa & $57,2 \mathrm{~b}$ & 57,0 & 98,0 & 24,0 & 0,1 & 0,6 & 21,0 \\
\hline \multirow{2}{*}{$\mathrm{NK}^{(3)}$} & Côncava & $124,1 \mathrm{~b}$ & 120,0 & 180,0 & 60,0 & 0,2 & $-1,5$ & 34,8 \\
& Convexa & $142,3 \mathrm{a}$ & 120,0 & 180,0 & 80,0 & $-0,3$ & $-1,4$ & 27,4 \\
\hline \multirow{2}{*}{$\mathrm{NC}^{(4)}$} & Côncava & $88,7 \mathrm{a}$ & 80,0 & 120,0 & 60,0 & 0,5 & $-1,1$ & 23,8 \\
& Convexa & $83,2 \mathrm{~b}$ & 80,0 & 120,0 & 60,0 & $-0,2$ & 0,9 & 23,7 \\
\hline & Côncava & $0,2 \mathrm{~b}$ & 0,0 & 0,8 & 0,0 & 1,3 & 0,2 & 146,7 \\
& Convexa & $0,3 \mathrm{a}$ & 0,2 & 1,3 & 0,0 & 1,1 & 0,4 & 119,1 \\
\hline
\end{tabular}

${ }^{(1)} \mathrm{V} \%$ - saturação por bases; ${ }^{(2)} \mathrm{NP}$ - necessidade de fósforo $\left(\mathrm{kg} \mathrm{ha}^{-1}\right) ;{ }^{(3)} \mathrm{NK}$ - necessidade de potássio $\left(\mathrm{kg} \mathrm{ha}^{-1}\right) ;{ }^{(4)} \mathrm{NC}$ - necessidade de calcário $\left(\mathrm{t} \mathrm{ha}^{-1}\right) ;{ }^{(5)}$ C.V.\% - coeficiente de variação. As médias das variáveis, acompanhadas da mesma letra, são, estatisticamente, iguais entre si, pelo teste t-Student, a $5 \%$ de probabilidade.

O fósforo e a necessidade de calagem apresentaram os maiores coeficientes de variação (C.V.\%), sendo superiores a $100 \%$ em ambas as formas de relevo. O manejo convencional e o 
intenso revolvimento do solo podem ser a causa desses elevados valores de CV\%. A alta variabilidade de fósforo pode ser atribuída a sua baixa mobilidade no solo e à aplicação deste nutriente em manejos convencionais.

Verificou-se maior necessidade de fósforo e calcário na área convexa e menor quantidade para área côncava, concordando com os resultados obtidos por BARBIERI et al. (2008). Para o potássio, a necessidade na área côncava foi maior, ocorrendo o inverso da situação para a necessidade de fósforo e calagem. Porém, o coeficiente de variação deste nutriente apresentou-se igual para as duas formas do relevo, indicando similaridade ou pouca variação nas quantidades aplicadas.

Todas as variáveis apresentaram dependência espacial (Tabela 2). O modelo que melhor se ajustou aos semivariogramas experimentais das variáveis foi o exponencial, o que mostra que estas variáveis têm comportamento mais errático na pequena escala, enquanto para os semivariogramas experimentais das variáveis magnésio, na pedoforma côncava e a necessidade de fósforo na área convexa, foi ajustado o modelo esférico, indicando comportamento menos errático na pequena escala. Alguns autores têm ajustado estes modelos para os atributos químicos do solo (CHANG et al., 2003; FRANZEN et al., 2006; SOUZA et al., 2006). GREGO \& VIEIRA (2005) verificaram que estes são os modelos que se ajustam com maior frequência aos atributos dos solos.

Conforme a classificação de CAMBARDELLA et al. (1994), a análise da relação $\mathrm{C}_{0} /\left(\mathrm{C}_{0}+\mathrm{C}_{1}\right)$ mostrou grau de dependência espacial moderado para todas variáveis em estudo, com exceção para a saturação por bases e a necessidade de calagem na área côncava (Tabela 2), que apresentaram grau de dependência alto e fraco, respectivamente, estando de acordo com os resultados deCHANG et al. (2003) e SOUZA et al. (2007).

Os valores de alcance para fósforo, cálcio, saturação por bases e necessidade de fósforo foram maiores na pedoforma convexa, o que indica menor continuidade espacial destas variáveis nesta forma de relevo, enquanto para potássio, magnésio, necessidade de calagem e necessidade de potássio, apresentaram valores de alcance maiores na área côncava, consequentemente menor continuidade espacial destas variáveis nesta pedoforma, concordando com os resultados observados por MONTANARI et al. (2005) e SOUZA et al. (2006).

TABELA 2. Coeficientes e modelos dos semivariogramas ajustados para os atributos químicos, necessidade de fósforo, potássio e calcário na profundidade de 0,00-0,20 m. Coefficients and adjusted semivariogram models for the chemical, the need for phosphorus, potassium and limestone at a depth of 0.00-0.20 m.

\begin{tabular}{|c|c|c|c|c|c|c|}
\hline Variável & Modelo & $\mathrm{C}_{0}^{(5)}$ & $\mathrm{C}_{0}+\mathrm{C}_{1}{ }^{(6)}$ & $\mathrm{C}_{0} /\left(\mathrm{C}_{0}+\mathrm{C}_{1}\right)^{(7)}$ & $a(m)^{(8)}$ & $\mathrm{R}^{2(9)}$ \\
\hline \multicolumn{7}{|c|}{ Côncava } \\
\hline $\mathrm{P}\left(\mathrm{mg} \cdot \mathrm{dm}^{-3}\right)$ & Exponencial & 251,64 & 536,99 & 0,47 & 121 & 0,20 \\
\hline $\mathrm{K}\left(\mathrm{mmol}_{\mathrm{c}} \cdot \mathrm{dm}^{-3}\right)$ & Exponencial & 0,30 & 0,67 & 0,44 & 546 & 0,96 \\
\hline $\mathrm{Ca}\left(\mathrm{mmol}_{\mathrm{c}} \cdot \mathrm{dm}^{-3}\right)$ & Exponencial & 11,15 & 29,29 & 0,38 & 239 & 0,59 \\
\hline $\mathrm{Mg}\left(\mathrm{mmol}_{\mathrm{c}} \cdot \mathrm{dm}^{-3}\right)$ & Esférico & 2,30 & 4,60 & 0,50 & 480 & 0,91 \\
\hline $\mathrm{V} \%^{(1)}$ & Exponencial & 15,10 & 114,00 & 0,13 & 141 & 0,77 \\
\hline $\mathrm{NP}^{(2)}$ & Exponencial & 958,98 & 1867,26 & 0,51 & 180 & 0,69 \\
\hline $\mathrm{NK}^{(3)}$ & Exponencial & 201,12 & 450,76 & 0,44 & 637 & 0,93 \\
\hline $\mathrm{NC}^{(4)}$ & Exponencial & 0,01 & 0,014 & 0,78 & 466 & 0,67 \\
\hline \multicolumn{7}{|c|}{ Convexa } \\
\hline $\mathrm{P}\left(\mathrm{mg} \cdot \mathrm{dm}^{-3}\right)$ & Esférico & 222,30 & 444,70 & 0,50 & 320 & 0,94 \\
\hline $\mathrm{K}\left(\mathrm{mmol}_{\mathrm{c}} \cdot \mathrm{dm}^{-3}\right)$ & Exponencial & 0,27 & 0,72 & 0,38 & 244 & 0,85 \\
\hline $\mathrm{Ca}\left(\mathrm{mmol}_{\mathrm{c}} \cdot \mathrm{dm}^{-3}\right)$ & Exponencial & 22,89 & 46,53 & 0,49 & 486 & 0,96 \\
\hline $\mathrm{Mg}\left(\mathrm{mmol}_{\mathrm{c}} \cdot \mathrm{dm}^{-3}\right)$ & Exponencial & 0,73 & 1,96 & 0,37 & 220 & 0,88 \\
\hline $\mathrm{V} \%^{(1)}$ & Exponencial & 53,60 & 117,40 & 0,46 & 345 & 0,92 \\
\hline $\mathrm{NP}^{(2)}$ & Esférico & 590,86 & 1476,45 & 0,40 & 305 & 0,74 \\
\hline $\mathrm{NK}^{(3)}$ & Exponencial & 169,83 & 338,60 & 0,50 & 209 & 0,62 \\
\hline $\mathrm{NC}^{(4)}$ & Exponencial & 0,04 & 0,11 & 0,35 & 207 & 0,77 \\
\hline
\end{tabular}


${ }^{(1)} \mathrm{V} \%$ - saturação por bases; ${ }^{(2)} \mathrm{NP}$ - necessidade de fósforo $\left(\mathrm{kg} \mathrm{ha}^{-1}\right) ;{ }^{(3)} \mathrm{NK}$ - necessidade de potássio $\left(\mathrm{kg} \mathrm{ha}^{-1}\right) ;{ }^{(4)} \mathrm{NC}$ - necessidade de calcário $\left(\mathrm{t} \mathrm{ha}^{-1}\right) ;{ }^{(5)} \mathrm{C}_{0}$ - efeito pepita; ${ }^{(6)} \mathrm{C}_{0}+\mathrm{C}_{1}$ - patamar; ${ }^{(7)} \mathrm{C}_{0} /\left(\mathrm{C}_{0}+\mathrm{C}_{1}\right)$ - grau de dependência espacial; ${ }^{(8)} \mathrm{a}$ - alcance; ${ }^{(9)} \mathrm{R}^{2}$ coeficiente de determinação.

Nas Figuras 2 e 3, são apresentados os mapas de variabilidade espacial para os atributos estudados e as recomendações de calagem e adubação.

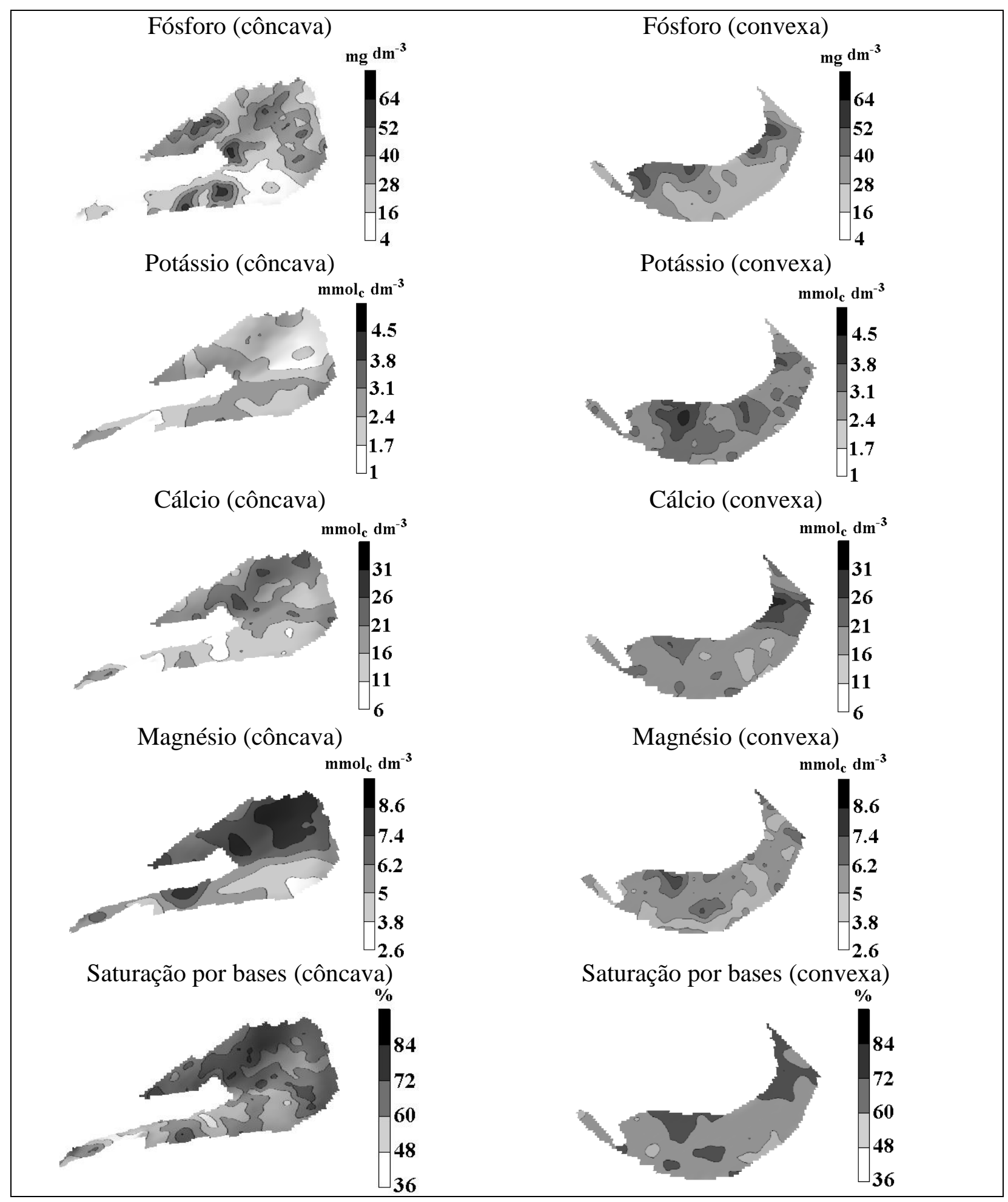

FIGURA 2. Mapas de krigagem para teores de fósforo, potássio, cálcio, magnésio e saturação por bases, na profundidade de 0,00-0,20 m. Kriging maps for phosphorus, potassium, calcium, magnesium and saturation at a depth of $0.00-0.20 \mathrm{~m}$. 


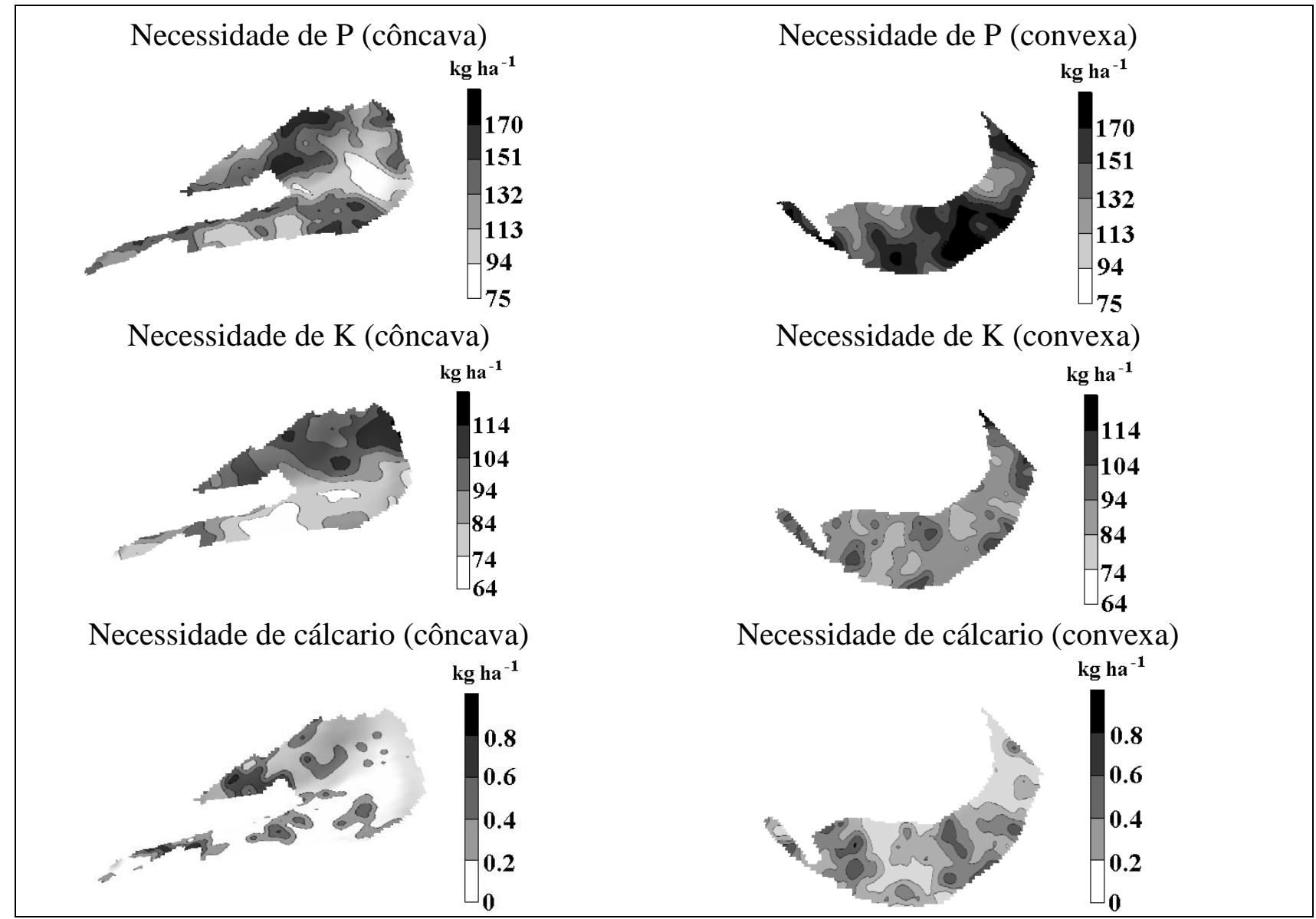

FIGURA 3. Mapas de krigagem para necessidade de aplicação de fósforo, potássio e calcário, na profundidade de $0,00-0,20 \mathrm{~m}$. Kriging maps for the application need of phosphorus, potassium and limestone at a depth of 0.00-0.20 m.

Analisando os mapas gerados por meio dos dados obtidos pelos semivariogramas (Figuras $2 \mathrm{e}$ 3), observaram-se menores distâncias entre as isolinhas da área côncava para as variáveis fósforo, cálcio, saturação por bases e necessidade de fósforo, demonstrando maior variabilidade nesta forma de relevo.

Nos mapas da área convexa, as distâncias foram maiores para as mesmas variáveis, mostrando comportamento inverso da área côncava. Estes resultados concordam com vários estudos que têm demonstrado a influência do relevo na variabilidade de atributos químicos do solo (CHANG et al., 2003; MONTANARI et al., 2005; FRANZEN et al., 2006; SOUZA et al., 2006).

Para o entendimento das causas da variabilidade do solo, é preciso conhecer os processos do solo que operam em locais específicos. Esses processos estão muito ligados ao fluxo de água que, por sua vez, são controlados pelo relevo (KRAVCHENKO \& BULLOCK, 2002). Neste sentido, a separação das formas do relevo mostra ser eficiente para identificação e mapeamento de áreas com variabilidade controlada, bem como a transferência de informações, podendo servir de base para elaboração e execução de programas de adubação (MALLARINO et al., 2001; SOUZA et al., 2007).

Do ponto de vista de aplicação de insumos, as doses recomendadas no mapa de necessidade de fósforo na área total variaram de 75 a $170 \mathrm{~kg} \mathrm{ha}^{-1}$ (Figura 3), levando em consideração a aplicação com taxa variada. Para a adubação convencional, fez-se a aplicação da média destes 
valores (123 kg ha ${ }^{-1}$ ), sendo que uma parte significativa da área recebe quantidade inferior, e outra, quantidade superior à dose necessária. WANG et al. (2006), KORSAETH \& RILEY (2006), e LAMBERT et al. (2006), estudando mapas de recomendação de manejo localizado, encontraram erros de dosagem para a adubação fosfatada.

Estas variações configuram-se nos mapas de necessidade de potássio e necessidade de calagem (Figura 3). No caso da necessidade de potássio, existe uma variação de recomendação de 65 a $112 \mathrm{~kg} \mathrm{ha}^{-1}$ e, com a média pelo método convencional, aplicaram-se $89 \mathrm{~kg} \mathrm{ha}^{-1}$. Para a necessidade de calagem, tem-se uma diferença de valores de 0,0-0,8 $\mathrm{t} \mathrm{ha}^{-1}$, sendo que, na média, deveria ser aplicado 0,4 $\mathrm{t} \mathrm{ha}^{-1}$. BARBIERI et al. (2008), em seus estudos, concluíram, baseados nos mapas de recomendação de calcário e fósforo, que grande parte da área não necessitaria de aplicação destes insumos, porém, no caso da agricultura convencional, haveria a recomendação de aplicação destes insumos.

Comparando-se os métodos da agricultura convencional, baseada na aplicação de insumos com taxa fixa, com a agricultura de precisão, com aplicação em taxas variadas, percebeu-se uma otimização significativa para a aplicação de insumos, quando se utiliza o método da agricultura de precisão. BIERMACHER et al. (2006) e LAMBERT et al. (2006) verificaram, em suas pesquisas, a economia de insumos agrícolas quando aplicaram a agricultura de precisão em relação à agricultura convencional.

Para a aplicação de calcário, observou-se que, na forma convencional, a dose deste insumo na área total foi de $500 \mathrm{t}$, e por meio da agricultura de precisão, a quantidade a ser utilizada foi de $64 \mathrm{t}$. Assim, boa parte da área recebe calcário de forma desnecessária, aumentando os custos de aplicação, causando inadequação ao equilíbrio na absorção de íons pelas plantas e um possível desequilíbrio ecológico na área de manejo convencional. BIERMACHER et al. (2006), estudando formas de adubação para cultura do trigo, concluíram que sistemas precisos podem reduzir o nível global de aplicação de nitrogênio entre 59-82\% em relação à aplicação convencional.

Em termos financeiros, a agricultura de precisão traria economia de calcário de aproximadamente US\$ 42,14 ha $^{-1}(89 \%)$ na área côncava, e de U\$ 39,23 ha ${ }^{-1}$ (83\%) na área convexa. Extrapolando esses valores para os 200 ha da área em estudo, chega-se ao custo total de calcário de US\$ 9.500,00 para a aplicação convencional, e de US\$1.380,11 para a aplicação com a taxa variada, o que proporciona a economia de US\$ 8.119,89 na área. BARBIERI et al. (2008) verificaram aplicações excessivas de calcário em áreas de aplicação convencional comparada com áreas de manejo localizado.

Para a aplicação de fertilizantes (Tabela 3), a quantidade da fórmula comercial de adubo (0412-10) a ser aplicada por meio do sistema convencional é de $200.000 \mathrm{~kg}$ nos 200 ha, sendo que, na aplicação por meio da agricultura de precisão, a quantidade de adubo na área total é reduzida para $85.636,61 \mathrm{~kg}$, devendo ser aplicados $38.807,80 \mathrm{~kg}$ na área côncava e 46.828,30 kg na área convexa.

Em termos financeiros, a economia da agricultura de precisão em relação ao sistema convencional para a aplicação de fertilizantes seria de US\$ 523,39 ha ${ }^{-1}(31,04 \%)$ para a área côncava e de US\$ 43,08 ha $^{-1}(25,10 \%)$ para a convexa. Considerando a área total de estudo, o custo de aplicação de fertilizantes por meio do sistema convencional foi de US\$ 34.400,00 enquanto a aplicação com taxa vairada foi de US\$ 11.163,57 na área côncava e de US\$13.650,05 na convexa, atingindo um total de US\$24.813,62 na área total. Desta forma, a aplicação de fertilizantes localizada proporcionará a economia de US\$ 9.586,38 na área estudada.

Os resultados para aplicação de insumos, com base nos dados dos pontos da malha de amostragem (agricultura de precisão), apresentaram valores de redução de 40,33\% dos custos de insumos, quando comparados com o sistema convencional de adubação (Tabela 3).

A área côncava apresentou maior fertilidade em relação à área convexa, condicionando custos de aplicação de US\$123,97 ha ${ }^{-1}$ e US\$ 137, 19 ha $^{-1}$, respectivamente. Portanto, existe a necessidade 
de adicionar $12 \%$ a mais de fertilizantes nos solos da área convexa. Observou-se, para o sistema de adubação convencional, que os custos para a aplicação de insumos é de U\$ 219,50 ha ${ }^{-1}$.

TABELA 3. Custo comparativo de adubação entre a aplicação em taxa fixa (AC) e variável (AP), sem e com a aplicação de modelagem matemática (MM) na área de 200 ha. Cost comparison between the fertilizer application rate (AC) and variable (AP), with and without the application of mathematical modeling (MM) in the area of 200 ha.

\begin{tabular}{|c|c|c|c|c|c|c|c|c|c|}
\hline \multirow{2}{*}{$\begin{array}{c}\text { Sistema de } \\
\text { adubação }\end{array}$} & \multirow{2}{*}{ Adubo } & \multicolumn{2}{|c|}{$\left(\mathrm{US} \$ \mathrm{~kg}^{-1}\right)$} & \multicolumn{2}{|c|}{ Aplicado kg) } & \multicolumn{2}{|c|}{ Dose $\left(\mathrm{kg} \mathrm{ha}^{-1}\right)$} & \multicolumn{2}{|c|}{$\left(\mathrm{US} \$ \mathrm{ha}^{-1}\right)$} \\
\hline & & $\mathrm{CM}^{(7)}$ & $\mathrm{SM}^{(8)}$ & $\mathrm{CM}$ & SM & $\mathrm{CM}$ & SM & $\mathrm{CM}$ & SM \\
\hline \multirow{6}{*}{ Taxa fixa } & $\mathrm{AA}^{(1)}$ & 0,460 & - & $3.658,50$ & - & 18,29 & - & 8,41 & \\
\hline & $\mathrm{FM}^{(2)}$ & 0,349 & - & $50.000,00$ & - & 250,00 & - & 87,25 & - \\
\hline & $\mathrm{CP}^{(3)}$ & 0,167 & - & $33.333,30$ & - & 166,67 & - & 27,83 & - \\
\hline & $\mathrm{CD}^{(4)}$ & 0,019 & - & $714.285,70$ & - & $3.571,43$ & & 67,85 & - \\
\hline & $F^{(5)}$ & - & 0,172 & - & 200.000 & - & 1.000 & - & 172,00 \\
\hline & $\mathrm{CC}^{(6)}$ & - & 0,019 & - & 500.000 & - & 2.500 & - & 47,50 \\
\hline Total AC & & & & & & & & 191,34 & 219,50 \\
\hline \multirow{14}{*}{$\begin{array}{c}\text { Taxa } \\
\text { variada }\end{array}$} & & \multicolumn{8}{|c|}{ Côncavo } \\
\hline & AA & \multirow{6}{*}{\multicolumn{2}{|c|}{$\begin{array}{l}0,460 \\
0,349 \\
0,167 \\
0,019 \\
0,023\end{array}$}} & 758,20 & 756,80 & 8,10 & 8,08 & 3,73 & 3,72 \\
\hline & FM & & & $24.222,70$ & $24.183,90$ & 258,73 & 258,32 & 90,30 & 90,15 \\
\hline & $\mathrm{CP}$ & & & $13.890,00$ & $13.867,10$ & 148,37 & 148,12 & 24,78 & 24,74 \\
\hline & $\mathrm{CD}$ & & & $4.976,40$ & $6.560,90$ & 53,16 & 70,08 & 1,01 & 1,96 \\
\hline & $\mathrm{CC}$ & & & $16.354,10$ & $13.851,10$ & 174,69 & 147,95 & 4,02 & 3,40 \\
\hline & $\underline{\text { Subtotal }}$ & & & & & & & 123,84 & 123,97 \\
\hline & & \multirow{2}{*}{\multicolumn{2}{|c|}{0,460}} & \multicolumn{4}{|c|}{ Convexo } & & \\
\hline & AA & & & 517,90 & 517,90 & 4,87 & 4,87 & 2,24 & 2,24 \\
\hline & FM & \multicolumn{2}{|c|}{0,349} & $31.533,70$ & $31.558,90$ & 296,42 & 296,66 & 103,45 & 103,53 \\
\hline & $\mathrm{CP}$ & \multicolumn{2}{|c|}{0,167} & $14.742,20$ & $14.751,50$ & 138,58 & 138,67 & 23,14 & 23,15 \\
\hline & $\mathrm{CD}$ & \multicolumn{2}{|c|}{0,019} & $26.711,00$ & $30.696,70$ & 251,09 & 288,56 & 4,77 & 5,48 \\
\hline & $\mathrm{CC}$ & \multirow{2}{*}{\multicolumn{2}{|c|}{0,023}} & $19.934,40$ & $12.890,60$ & 187,39 & 121,17 & 4,31 & 2,79 \\
\hline & Subtotal & & & & & & & 137,91 & 137,19 \\
\hline Total AP & & & & & & & & $131,29 *$ & $130,97 *$ \\
\hline
\end{tabular}

Extrapolando-se estes valores para a área total, verificou-se que o sistema de adubação convencional apresentou um custo de aplicação de insumos (calcário e fertilizante) de US\$ 43.900,00, enquanto a aplicação de insumos por meio da agricultura de precisão apresentou um custo total de US\$ 26.193,74 (US\$ 11.668,06 na área côncava e 14.525,68 na convexa), possibilitando a economia de US\$ 17.706,26 na área de estudo. MOLIN et al.(2006), trabalhando com unidades de aplicação com diferentes necessidades para o fósforo, observaram redução de $14 \%$ na demanda desse nutriente.

Observou-se que tanto a aplicação de calcário como a de fertilizante apresentaram as maiores doses para a área convexa, em relação à área côncava, demonstrando a eficiência da caracterização das formas da paisagem para o melhor rendimento da agricultura de precisão. Neste sentido, a separação das formas do relevo mostra ser eficiente para identificação e mapeamento de áreas com variabilidade controlada, bem como a transferência de informações, podendo servir de base para elaboração e execução de programas de adubação (MALLARINO et al., 2001).

Os dados da adubação convencional, inseridos no modelo matemático (MM), apresentaram redução de custo de $13 \%$ na aplicação de calcário e fertilizante em relação ao uso de formulado e cálcário, proporcionando a economia de US\$ 5.632,00 (Tabela 3). O custo para aplicação de adubo com base nos dados dos pontos da malha (agricultura de precisão), inseridos no modelo matemático, apresentou redução de 40,19\% em relação ao método convencional sem modelagem matemática, indicando ser a associação destas técnicas mais eficiente do ponto de vista econômico. 
A utilização de modelos matemáticos pode auxiliar no planejamento da produção de usinas sucroalcooleiras, proporcionando agilidade, facilidade e confiabilidade nas análises realizadas, propiciando análises quantitativas eficazes que apoiam o planejamento de safra e a melhoria nos resultados financeiros da empresa (PAIVA \& MORABITO, 2007). A associação dos modelos matemáticos com a técnica geoestatística e com os conceitos da relação solo-geomorfologia traz grandes beneficios para o planejamento agrícola com base na variabilidade dos atributos do solo em escala detalhada (zona de manejo específico). A limitação deste tipo de mapeamento em escala detalhada é justamente o número de amostras necessárias para a caracterização da variabilidade espacial. Assim, segundo SIQUEIRA et al. (2010b), trabalhos futuros que vislumbrem o mapeamento dos atributos do solo em escalas mais detalhadas, devem refletir a possibilidade de uso de técnicas de quantificação indireta, como a suscetibilidade magnética do solo (SM). De acordo com estes autores, a SM teve uma correlação de 0,6 com V\%. Dessa maneira, pode-se inferir que a SM pode ser utilizada para auxiliar na recomendação da necessidade de calagem, adubação e outras práticas agrícolas.

\section{CONCLUSÕES}

Os limites das formas do relevo são fortes indicadores das zonas específicas para aplicação de insumos à taxa variada.

Os mapas gerados para aplicação de insumos à taxa variável demonstraram ser mais eficientes do ponto de vista econômico e ambiental do que aplicações baseadas na média.

A interação do modelo matemático e a agricultura de precisão apresentaram maior eficiência na redução de custos para fertilização de áreas agrícolas, cultivadas com cana-de-açúcar.

\section{REFERÊNCIAS}

BARBIERI, D.M.; MARQUES JÚNIOR, J.; PEREIRA, G.T. Variabilidade espacial de atributos químicos de um argissolo para aplicação de insumos à taxa variável em diferentes formas de relevo. Engenharia Agrícola, Jaboticabal, v.28, n.4, p.645-653, 2008.

BIERMACHER, J.T.; EPPLIN, F.M.; BRORSEN, B.W.; SOLIE, J.B.; RAUN, W.R. Maximum benefit of a precise nitrogen application system for wheat. Precision Agriculture, Dordrecht, v.7, n.3, p.193-204, 2006.

BISHOP, T.F.A.; McBRATNEY, A.B. Creating field extent digital elevation models for precision agriculture. Precision Agriculture, Dordrecht, v.3, n.1, p.37-46, 2002.

CAMBARDELLA, C.A.; MOORMAN, T.B.; NOVAK, J.M.; PARKIN, T.B.; KARLEN, D.L.; TURCO, R.F.; KONOPKA, A.E. Field-scale variability of soil properties in central Iowa soils. Soil Science Society of America Journal, Madison, v.58, n.5, p.1.501-1.511, 1994.

CHANG, J.; CLAY, D.E.; CARLSON, C.G.; CLAY, S.A.; MALO, D.D.; BERG, R.; KLEINJAN, J.; WIEBOLD, W. Different techniques to identify management zones impact nitrogen and phosphorus sampling variability. Agronomy Journal, Madison, v.95, p.1.550-1.559, 2003.

DODERMANN, A.; PING, J. L. Geostatistical integration of yield monitor data and remote sensing improves yield maps. Agronomy Journal, Madison, v.96, n.1, p.285-297, 2004.

EMBRAPA. EMPRESA BRASILEIRA DE PESQUISA AGROPECUÁRIA. Centro Nacional de Pesquisa de Solos. Sistema brasileiro de classificação de solos. Brasília, 2006. 306 p.

FRANZEN, D.W.; NANNA, T.; NORVELL, W.A. A survey of soil attributes in North Dakota by landscape position. Agronomy Journal, Madison, v.98, n.4, p.1.015-1.022, 2006.

GREGO, C.R.; VIEIRA, S.R. Variabilidade espacial de propriedades físicas do solo em uma parcela experimental. Revista Brasileira de Ciência do Solo, Viçosa-MG, v.29, n.2, p.169-177, 2005. 
IANNONI, A. P.; MORABITO. R. A discrete simulation analysis of a logistics supply system. Transportation Research Part E: Logistics and Transportation Review, Vancouver, v.42, n.3, p.191-210, 2006.

IPT. INSTITUTO DE PESQUISAS TECNOLÓGICAS DO ESTADO DE SÃO PAULO. Mapa geomorfológico do Estado de São Paulo. Escala - 1:1.000.000. São Paulo: IPT, 1981. v.1, 94 p.

KASPAR, T.C.; COLVIN, T.S.; JAYNES, D.B.; KARLEN, D.L.; JAMES, D.E.; MEEK, D.W. Relationship between six years of corn yields and terrain attributes. Precision Agriculture, Dordrecht, v.4, n.1, p.87-101, 2003.

KAWAMURA, M.S.; RONCONI, D.P.; YOSHIZAKI, H. Optimizing transportation and storage of final products in the sugar and ethanol industry. International Transactions in Operational Research, Oxford, v.13, n.5, p.425-439, 2006.

KORSAETH, A.; RILEY, H. Estimation of economic and environmental potentials of variable rate versus uniform $\mathrm{N}$ fertilizer application to spring barley on morainic soils in se norway. Precision Agriculture, Dordrecht, v.7, n.4, p.265-279, 2006.

KRAVCHENKO, A.N.; BULLOCK, D.G. Spatial variability of soybean quality data as a function of field topography: i spatial data analysis. Crop Science, Madison, v.42, n.3, p.804-815, 2002.

LAMBERT, D.M.; LOWENBERG-DEBOER, J.; MALZER, G.L. Economic analysis of spatialtemporal patterns in corn and soybean response to nitrogen and phosphorus. Agronomy Journal, Madison, v.98, n.1, p.43-54, 2006.

LANDI, A.; MERMUT, A.R.; ANDERSON, D.W. Carbon distribution in a hummocky landscape from Saskatchewan, Canada. Soil Science Society of America Journal, Madison, v.68, n.1, p.175-184, 2004.

MALLARINO, A.P.; MAZHAR, U.H.; WITTRY, D.; BERMUDEZ, M. Variation in soybean response to early season foliar fertilization among and within fields. Agronomy Journal, Madison, v.93, n.6, p.1.220-1.226, 2001.

MARTÍN, N.F.; BOLLERO, G.A.; BULLOCK, D.G. Associations between field characteristics and soybean plant performance using canonical correlation analysis. Plant Soil, The Hague, v.273, n.1-2, p.39-55, 2005.

MOLIN, J.P.; MASCARIN, L.S.; VIEIRA JÙNIOR, P.A. Avaliação de intervenções em unidades de aplicação localizada de fertilizantes e de populações de milho. Engenharia Agrícola, Jaboticabal, v.26, n.2, p.528-536, 2006.

MONTANARI, R.; MARQUES JÚNIOR, J.; PEREIRA, G. T.; SOUZA, Z.M. Forma da paisagem como critério para otimização amostral de Latossolos sob cultivo de cana-de-açúcar. Pesquisa Agropecuária Brasileira, Brasília, v.40, n.1, p.69-77, 2005.

PAIVA, R.P.O.; MORABITO, R. Um modelo de otimização para o planejamento agregado da produção em usinas de açúcar e álcool. Gestão e Produção, São Carlos, v.14, n.1, p.25-41, 2007.

RAIJ, van B.; CANTARELLA, H.; QUAGGIO, J.A. Análise química para avaliação da fertilidade de solos tropicais. Campinas: Instituto Agronômico, 2001. 285 p.

RAIJ, van B.; CANTARELLA, H.; QUAGGIO, J.A.; FURLANI, A.M.C. Recomendações de adubação e calagem para o estado de São Paulo. 2.ed. Campinas: Instituto Agronômico/Fundação IAC, 1997. 285 p. (Boletim Técnico, 100).

ROBERTSON, G.P. GS $S^{+}$geostatistics for the environmental sciences: $G S^{+}$user' guide. Plainwell: Gamma Design Software, 1998. 152 p.

SCHLOTZHAVER, S.D.; LITTELL, R.C. SAS: system for elementary statistical analysis. $2^{\text {nd }}$ ed. Cary: SAS, 1997. 905 p. 
SI, B.C.; FARRELL, R.E. Scale-dependent relationships between wheat yield and topographic indices: a wavelet approach. Soil Science Society of America Journal, Madison, v.68, n.2, p.577$588,2004$.

SINGH, N.; KAR, A. Characteristics of major soils of Banni mudflat in arid western India and their relationship with topography. Journal of Arid Environments, London, v.48, n.2, p.509-520, 2001.

SIQUEIRA, D. S.; MARQUES JR., J.; MATIAS, S.S.R.; BARRÓN, V.; TORRENT, J.; BAFFA, O.; OLIVEIRA, L.C. de. Predicting the properties of Brazilian Haplustalfs from magnetic susceptibility measurements. Soil Use and Management, Oxford, v.26, 425-431, $2010 \mathrm{~b}$.

SIQUEIRA, D.S.; MARQUES JR., J.;PEREIRA, G.T. The use of landforms to predict the variability of soil and orange attributes. Geoderma, Amsterdam, v. 155, p. 55-66, 2010a.

SOUZA, Z.M.; BARBIERI, D.M.; MARQUES JÚNIOR, J.; PEREIRA, G.T.; CAMPOS, M.C. Influência da variabilidade espacial de atributos químicos de um Latossolo na aplicação de insumos para a cultura de cana-de-açúcar. Ciência e Agrotecnologia, Lavras, v.31, n.2, p.371-377, 2007.

SOUZA, Z.M.; MARQUES JÚNIOR, J.; PEREIRA, G.T.; BARBIERI, D.M. Small relief shape variations influence spatial variability of soil chemical attributes. Scientia Agricola, Piracicaba, v.63, n.2, p.161-168, 2006.

SURFER. Surfer 7.0 Contouring and 3D surgaces mapping for scientist's engineers. Version 7.0. User's Guide. New York: Golden Software, 1999. 619 p.

TROEH, F.R. Landform equations fitted to contour maps. American Journal of Science, New Haven, v.263, n.3, p.616-627, 1965.

VIEIRA, S.R.; HATFIELD, J.L.; NIELSEN, D.R.; BIGGAR, J.W. Geostatistical theory and application to variability of some agronomical properties. Hilgardia, Berkeley, v.51, p.1-75, 1983.

WANG B.H.; JIN, J.; WANG, B. Improvement of soil nutrient management via information technology. Better Crops, Atlanta, v.90, n.3, p.30-32, 2006.

WEBSTER, R. Is soil variation random? Geoderma, Amsterdam, v.97, n.3, p.149-163, 2000. 